PROCEEDINGS OF THE

AMERICAN MATHEMATICAL SOCIETY

Volume 137, Number 7, July 2009, Pages 2175-2180

S 0002-9939(08)09745-1

Article electronically published on December 18, 2008

\title{
A REMARK ON MULTIVALUED ALGEBRAIC GROUPS
}

\author{
ANAND PILLAY
}

(Communicated by Julia Knight)

\begin{abstract}
We point out how suitable algebraic $n$-valued groups (in the sense of Buchstaber) give rise, in a reasonably canonical manner, to algebraic groups. This is proved using the "group configuration theorem" of Hrushovski. In particular this applies to all algebraic 2-valued groups.
\end{abstract}

\section{INTRODUCTION AND STATEMENT OF RESULTS}

Given a set $X,(X)^{n}$ denotes the family of $n$-element subsets of $X$, modified so that we allow elements to have multiplicity $>1$. So a typical element of $(X)^{n}$ could be written as a formal sum $k_{1} x_{1}+\cdots+k_{r} x_{r}$ or as $\left\{k_{1} x_{1}, \ldots, k_{r} x_{r}\right\}$ where $x_{1}, \ldots, x_{r}$ are distinct elements of $X$ and $k_{1}, \ldots, k_{r}$ are positive integers whose sum is $n$. We will say that $y \in(X)^{n}$ contains $x \in X$ if $x$ appears in $y$ with multiplicity $\geq 1$.

The notion of an $n$-valued group developed from work of Buchstaber and Novikov in the 1970's. Soon afterwards Buchstaber developed the theory of formal, or local, $n$-valued Lie groups and introduced the notion of an $n$-valued algebraic group. Since 1993 Buchstaber and E. Rees have collaborated on the topological and algebraic theory of $n$-valued groups. As defined in [1] an $n$-valued group is a set $X$ equipped with a distinguished element $e$ and operations $*$ from $X \times X$ to $(X)^{n}$ and $i n v$ from $X$ to $X$ such that:

(i) $x *(y * z)=(x * y) * z$ for any $x, y, z \in X$,

(ii) $e * x=x * e=n x$ for any $x \in X$,

(iii) both $x * \operatorname{inv}(x)$ and $i n v(x) * x$ contain $e$.

Possibly (i) (associativity) needs some explanation: by $x *(y * z)$ we mean the obvious element of $(X)^{n^{2}}$, and likewise for $(x * y) * z$. So (i) says that these elements of $(X)^{n^{2}}$ are equal.

Note that a 1-valued group $(G, \cdot, i n v)$ is the same thing as a group, and can also be considered as an $n$-valued group $(G, *)$ by writing $a * b=n(a \cdot b)$.

There are reasonably obvious notions of an algebraic $n$-valued group. For example, let $X$ be a quasiprojective variety. Then $(X)^{n}=X^{n} / S_{n}$ (where $S_{n}$ is the symmetric group acting naturally on $X^{n}$ ) has also naturally the structure of an algebraic variety. So we could view an "algebraic $n$-valued group" as a quasiprojective variety, equipped with morphisms $\mu: X \times X \rightarrow(X)^{n}$ and inv $: X \rightarrow X$

\footnotetext{
Received by the editors December 25, 2007, and, in revised form, July 22, 2008, and August 29, 2008.

2000 Mathematics Subject Classification. Primary 14L10; Secondary 03C45.

The author was supported by a Marie Curie Chair EXC 024052 as well as EPSRC grant
} $\mathrm{EP} / \mathrm{F} 009712 / 1$.

(C)2008 American Mathematical Society Reverts to public domain 28 years from publication 
and a distinguished element $e$ such that writing $\mu(x, y)$ as $x * y,(X, *, i n v, e)$ is an $n$-valued group. Alternatively we could consider an arbitrary variety $X$ and view the operation $*$ as given by a subvariety $\Gamma$ of $X \times X \times X$; namely, for $a, b \in X$, $\Gamma(a, b, z)$ is a 0 -dimensional subvariety of $X$ whose set of points, counted with multiplicities, is precisely $a * b$. Our results will hold with either definition, although we will be assuming that the underlying variety $X$ is absolutely irreducible.

From now on, $(X, *, i n v, e)$ is an irreducible algebraic $n$-valued group, defined over (in the obvious sense) a field $k$, and we will identify $X$ with its set $X(K)$ of $K$-points for some algebraically closed field $K$ of infinite transcendence degree over $k$. Let $m=\operatorname{dim}(X)$. We will assume $k$ to be algebraically closed.

We will also be assuming:

(iv) inv is generically finite-to-one, or equivalently the regular map inv :X $\rightarrow X$ is dominant (the image of $i n v$ is Zariski dense).

For $A$ any finite set of finite tuples from $K$, by $\operatorname{dim}(A / k)$ we mean the transcendence degree of $k(A)$ over $k$. A point $a \in X(K)$ is said to be generic over $k$ if $\operatorname{dim}(a / k)=m$ (the dimension of the algebraic variety $X$ ). A similar statement can be made for mutually generic, etc.

We will say that $X$ is generically of type $\left(k_{1}, \ldots, k_{r}\right)$ if for some, equivalently any, $a, b \in X$, which are mutually generic over $k, a * b=\left\{k_{1} x_{1}, \ldots, k_{r} x_{r}\right\}$ (with the $x_{i}$ distinct). Our main result is:

Proposition 1.1. Suppose that $X$ is generically of type $(1,1, \ldots, 1)$. Then there is a connected algebraic group $\left(G, \cdot, e^{\prime}\right)$ of dimension $m$, and a constructible subset $R$ of $X \times G$, such that

(a) $R$ projects dominantly onto both $X$ and $G$,

(b) for any $x \in X$ there are at most finitely many $g \in G$ such that $(x, g) \in R$ and dually (so in particular the dimension of the Zariski closure of $R$ is $m$ ),

(c) $\left(e, e^{\prime}\right) \in R$,

(d) for mutually generic $(x, g)$ and $(y, h)$ in $R$, there is $z \in x * y$ such that $(z, g \cdot h) \in R$

(e) for generic $(x, g) \in R,\left(\operatorname{inv}(x), g^{-1}\right) \in R$.

Proposition 1.1 is proved in section 3 using a straightforward lemma (3.1) about $n$-valued algebraic groups of type $(1,1, \ldots, 1)$ and some model theory, more precisely the "group configuration theorem". Lemma 3.1 holds trivially if $X$ is of type $(n)$; hence the conclusion of Proposition 1.1 holds for arbitrary 2-valued algebraic groups.

It is not hard to see that if $G_{1}$ is another connected algebraic group satisfying the conclusion of Proposition 1.1 (with, say, $R_{1}$ in place of $R$ ), then $G$ and $G_{1}$ will be isogenous.

If $G$ is a connected algebraic group and $A$ is a finite group of (rational) automorphisms of $G$ of cardinality $n$, then one obtains an $n$-valued algebraic group structure on $X=G / A$ in the obvious way. This is called a coset group by Victor Buchstaber; note that it is generically of type $(1,1, \ldots, 1)$. So Proposition 1.1 applies to $X$ and, by what we have said above, recovers $G$ up to isogeny.

It would be natural for a model-theorist to try to study "definable" multivalued groups in arbitrary stable theories, and formulate the results of this paper at a greater level of generality. Our definitions and proofs adapt quite straightforwardly to the context of $\omega$-stable theories. We leave further generalizations to the interested reader. 
We have made an effort to make the paper accessible to non-model-theorists. So we will use mainly the language of algebraic geometry at least in the sense of André Weil. We will also make some use of the language of model theory, and (as suggested by the referee) in section 2 we give explanations and translations of these notions, as well as stating the group configuration theorem in the context we need it.

Thanks to Victor Buchstaber for suggesting that these observations be written up for publication.

\section{Preliminaries}

We start by repeating a few notions from the introduction. As there, $k<K$ will be algebraically closed fields such that $K$ has infinite transcendence degree over $k$, and for $X$ an irreducible algebraic variety defined over $k$ we identify $X$ with its set $X(K)$ of $K$-rational points. We will usually be able to work in a given affine open subset of $X$ defined over $k$, so identified with a subset of $K^{n}$. So we will speak of points of $X$ as points $a \in K^{n}$ (for suitable $n$ ). We call $a$ a generic point of $X$ over $k$ if $\operatorname{dim}(a / k)=_{\operatorname{def}} \operatorname{tr} \cdot \operatorname{deg}(k(a) / k)=\operatorname{dim}(X)$. By the irreducibility of $X$, for any generic points $a, b$ of $X$ over $k$ there is $f \in A u t(K / k)$ (automorphisms of $K$ fixing $k$ pointwise) such that $f(a)=b$. We say here that $a$ and $b$ have the same type over $k$. In fact, we will take the following as a definition: for any finite tuple $a$ from $K$, the type of $a$ over $k$ (written $t p(a / k)$ ) is the orbit of $a$ under $A u t(K / k)$, and a realization of $t p(a / k)$ is just a point in that orbit.

A set $\left\{a_{1}, \ldots, a_{r}\right\}$ of tuples from $K$ is said to be independent over $k$ if $\operatorname{dim}\left(a_{1} a_{2} \ldots a_{r} / k\right)=\operatorname{dim}\left(a_{1} / k\right)+\cdots+\operatorname{dim}\left(a_{r} / k\right)$. Given an irreducible variety $X$ over $k$, by mutually generic points of $X$ over $k$ we mean generic points $a, b$ of $X$ over $k$ which are also independent over $k$.

Recall that a constructible subset of $K^{n}$ is a finite union of locally Zariski closed subsets of $K^{n}$, and likewise for a constructible subset of a variety. It makes sense to speak of a constructible set being defined over $k$.

An important notion: definability of types from stability theory says, in the special case of the theory of algebraic closed fields:

Fact 2.1. Let $R \subset K^{n+m}$ be constructible over $k$. Let $a \in K^{m}$ and $p=t p(a / k)$. Then $S=\left\{c \in K^{n}\right.$ : for some (any) $a^{\prime}$ realizing $p$ which is independent from $c$ over $k,(c, a) \in R\}$ is constructible over $k$.

Actually, Fact 2.1 is easy to prove algebraic-geometrically. It is enough to deal with the special case where $R$ is itself a subvariety of $K^{n+m}$. Let $R_{a} \subseteq K^{n}$ be the variety $\left\{x \in K^{n}:(x, a) \in R\right\}$. Let $p=t p(a / k)$. Then it is easy to check that $S=\bigcap\left\{R_{a^{\prime}}: a^{\prime}\right.$ realizes $\left.p\right\}$, which is a variety by Noetherianity of the Zariski topology and is clearly defined over $k$.

We will now state the group configuration theorem of Hrushovski in our context. We refer the interested reader to Theorem 5.4.5 of [2] for the most general modeltheoretic statement and proof.

Definition 2.2. A group configuration (in $K$ over $k$ ) consists of a set $\{a, b, c, x, d, f\}$ of finite tuples from $K$ and distinguished "lines" $\{a, b, c\},\{a, x, d\},\{x, b, f\},\{d, f, c\}$ such that

(i) each noncollinear triple from $\{a, b, c, x, d, f\}$ is independent over $k$, 
(ii) each element of each collinear triple is in the algebraic closure of the other two (so, for example, considering the collinear triple $\{a, b, c\}$ this says that $a \in k(b, c)^{a l g}$, $b \in k(a, c)^{a l g}$ and $\left.c \in k(a, b)^{a l g}\right)$.

Note that the definition implies that $\operatorname{dim}(a / k)=\operatorname{dim}(b / k)=\cdots=\operatorname{dim}(d / k)$.

Proposition 2.3. Let $\{a, b, c, x, d, f\}$ be a group configuration in $K$ over $k$. Then there is a connected algebraic group $(G, \cdot)$ defined over $k$ and generic points $a^{\prime}, b^{\prime}, c^{\prime}$ of $G$ over $k$ such that $a^{\prime} \cdot b^{\prime}=c^{\prime}$ and $k(a)^{\text {alg }}=k\left(a^{\prime}\right)^{\text {alg }}, k(b)=k\left(b^{\prime}\right)^{\text {alg }}$ and $k(c)=k\left(c^{\prime}\right)^{a l g}$.

\section{Proofs}

An important ingredient is the following:

Lemma 3.1. Under the assumptions of Proposition 1.1 ( $X$ is an irreducible $m$ dimensional algebraic $n$-valued group over $k$ of type $(1, \ldots, 1)$ satisfying also (iv)), let $a, b \in X$ be mutually generic over $k$, and let $c \in a * b$. Then

(i) $a \in c * i n v(b), b \in \operatorname{inv}(a) * c$, and

(ii) $\operatorname{dim}(a, b, c / k)=\operatorname{dim}(a, b / k)=\operatorname{dim}(b, c / k)=\operatorname{dim}(a, c / k)=2 m$.

Proof. Let $a * b=\left\{c_{1}, \ldots, c_{n}\right\}$. Consider $a *(b * i n v(b))$. It contains $a * e=n a$ ( $a$ with multiplicity $n$ ). By associativity $a$ appears with multiplicity at least $n$ in $(a * b) * i n v(b)$. So $a \in c_{i} * i n v(b)$ for some $i=1, \ldots, n$. By assumption (iv), $k(b)^{a l g}=$ $k(\operatorname{inv}(b))^{a l g}$; hence $k(a, b)^{a l g}=k\left(c_{i}, i n v(b)\right)^{a l g}$ whereby $\operatorname{dim}\left(c_{i}, i n v(b) / k\right)=2 m$ from which it follows that $c_{i}$ and $i n v(b)$ are mutually generic (over $k$ ) elements of $X$. So $a$ appears with multiplicity 1 in $c_{i} * i n v(b)$. Hence $a \in c_{j} * i n v(b)$ for some $j \neq i$. Repeating the argument, we conclude that $c_{j}$ and $i n v(b)$ are mutually generic and $a$ appears with multiplicity 1 in $c_{j} * i n v(b)$. Continuing (using the fact that $n a \in(a * b) *(i n v(b)))$ we conclude that for each $i=1, \ldots, n, a \in c_{i} * i n v(b)$ and $c_{i}$ and $i n v(b)$ are mutually generic elements of $X$.

Considering instead $(\operatorname{inv}(a) * a) * b$ and repeating the argument, we see that for each $i=1, \ldots, n, b \in \operatorname{inv}(a) * c_{i}$, and $c_{i}$ and $i n v(a)$ are mutually generic.

So we obtain (i), and using also that $k(a)^{a l g}=k(i n v(a))^{a l g}$ and $k(b)=$ $k(i n v(b))^{a l g}$ we obtain (ii). Lemma 3.1 is proved.

We now prove Proposition 1.1. Let $a, b$ be mutually generic in $X$ over $k$ and let $c \in a * b$. The first step is to recover from $X$ the "group configuration". Let $x \in X$ be chosen generic over $k(a, b)$. By associativity $a *((x * i n v(x)) * b)=(a * x) *(i n v(x) * b)$. Now $c$ is on the left-hand side. So there are $d \in a * x$ and $f \in i n v(x) * b$ such that $c \in d * f$. Our configuration consists of the points $a, b, c, x, d, f$ and the "lines" $\{a, b, c\},\{a, x, d\},\{x, b, f\},\{d, f, c\}$. Note that $k(x)^{a l g}=k(\text { inv }(x))^{\text {alg }}$ (by (iv)).

By Lemma 3.1, we have

$\left(^{*}\right)$ each noncollinear triple from $\{a, b, c, x, d, f\}$ is independent over $k$, and each element of each collinear triple is in the algebraic closure of the other two.

By the group configuration theorem, Proposition 2.3, we have

Lemma 3.2. There is a connected $m$-dimensional algebraic group $\left(G, \cdot,{ }^{-1}, e^{\prime}\right)$ defined over $k$ and generic points $a^{\prime}, b^{\prime}, c^{\prime}$ of $G$ over $k$ such that $a^{\prime} \cdot b^{\prime}=c^{\prime}$ and $k(a)^{\text {alg }}=k\left(a^{\prime}\right)^{\text {alg }}, k(b)^{\text {alg }}=k\left(b^{\prime}\right)^{\text {alg }}$ and $k(c)^{\text {alg }}=k\left(c^{\prime}\right)^{\text {alg }}$.

We now find the $R$ from Proposition 1.1. We can find an automorphism $\alpha$ of $K$ over $k$ which fixes $\left(c, c^{\prime}\right)$ and takes $\left(a, a^{\prime}\right)$ to $\left(a_{1}, a_{1}^{\prime}\right)$, where $\left(a_{1}, a_{1}^{\prime}\right)$ is independent 
from $\left(a, a^{\prime}, b, b^{\prime}, c, c^{\prime}\right)$ over $k$. (Namely, $\operatorname{dim}\left(a_{1}, a_{1}^{\prime} / k\left(a, a^{\prime}, b, b^{\prime}, c, c^{\prime}\right)\right)=m$.) Let $\left(b_{1}, b_{1}^{\prime}\right)=\alpha\left(b, b^{\prime}\right)$.

So

(a) $a^{\prime} \cdot b^{\prime}=c^{\prime}$ and $a_{1}^{\prime} \cdot b_{1}^{\prime}=c^{\prime}$; hence $\left(\left(a_{1}^{\prime}\right)^{-1} \cdot a^{\prime}\right) \cdot b^{\prime}=b_{1}^{\prime}$.

Put $a_{2}^{\prime}=\left(a_{1}^{\prime}\right)^{-1} \cdot a^{\prime}$.

Also

(b) $c \in a * b$ and $c \in a_{1} * b_{1}$.

By Lemma 3.1, $b_{1} \in \operatorname{inv}\left(a_{1}\right) * c$, so by associativity there is $a_{2} \in \operatorname{inv}\left(a_{1}\right) * a$ such that $b_{1} \in a_{2} * b$.

So we have

(c) $b_{1}^{\prime}=a_{2}^{\prime} \cdot b^{\prime}$ and $b_{1} \in a_{2} * b$.

Lemma 3.3. $\operatorname{dim}\left(a_{2} / k\right)=\operatorname{dim}\left(a_{2}^{\prime} / k\right)=\operatorname{dim}\left(a_{2}, a_{2}^{\prime} / k\right)=m$, and $\left(a_{2}, a_{2}^{\prime}\right)$ is independent from each of $\left(b, b^{\prime}\right)$ and $\left(b_{1}, b_{1}^{\prime}\right)$ over $k$.

Proof. It is clear from Lemma 3.1 that $\operatorname{dim}\left(a_{2} / k\right)=\operatorname{dim}\left(a_{2}^{\prime} / k\right)=m$. Now $\left(a_{2}, a_{2}^{\prime}\right) \in k\left(a, a^{\prime}, a_{1}, a_{1}^{\prime}\right)^{a l g}$ and $\left(a, a^{\prime}, a_{1}, a_{1}^{\prime}\right)$ is independent from $\left(b, b^{\prime}\right)$ over $k$. So $\left(a_{2}, a_{2}^{\prime}\right)$ is independent from $\left(b, b^{\prime}\right)$ over $k$. But $\left(a_{2}, a_{2}^{\prime}\right) \in k\left(b, b^{\prime}, b_{1}, b_{1}^{\prime}\right)^{a l g}$, and $\operatorname{dim}\left(b_{1}, b_{1}^{\prime} / k\left(b, b^{\prime}\right)\right)=m$. This forces $\operatorname{dim}\left(a_{2}, a_{2}^{\prime} / k\right)$ to be at most $m$, hence exactly $m$. The rest of the lemma follows.

Let $V \subseteq X \times G$ be the irreducible algebraic variety over $k$ whose generic point is $\left(b, b^{\prime}\right)$. So $\left(\right.$ as $\left.\left(b_{1}, b_{1}^{\prime}\right)=\alpha\left(b, b^{\prime}\right)\right)\left(b_{1}, b_{1}^{\prime}\right)$ is also a generic point of $V$ over $k$. Note that $\operatorname{dim}(V)=m$. Using the model-theoretic notation explained in section 2, let $p=t p\left(b, b^{\prime} / k\right)$, the "generic type" of $V$, and so the realizations of $p$ are precisely the generic points (over $k$ ) of $V$. $R$ will be a kind of "stabilizer" of $V$ or $p$.

More precisely let $R \subset X \times G$ be the set of $\left(y, y^{\prime}\right)$ such that for some (any) realization $\left(d, d^{\prime}\right)$ of $p$ independent from $\left(y, y^{\prime}\right)$ over $k$ there is $\left(d_{1}, d_{1}^{\prime}\right)$ realizing $p$ such that $d_{1}^{\prime}=y^{\prime} \cdot d^{\prime}, d_{1} \in y * d, d \in i n v(y) * d_{1}$ and $y \in d_{1} * i n v(d)$. By Fact 2.1 $R$ is constructible over $k$.

Lemma 3.4. (i) $\left(a_{2}, a_{2}^{\prime}\right) \in R$.

(ii) For any $y \in X$ there are at most finitely many $y^{\prime} \in G$ such that $\left(y, y^{\prime}\right) \in R$, and dually. (Hence using (i), $R$ has dimension $m$.) $\in R$.

(iii) If $\left(y, y^{\prime}\right) \in R$ is generic over $k$, namely $\operatorname{dim}\left(y, y^{\prime} / k\right)=m$, then $\left(\operatorname{inv}(y), y^{-1}\right)$

(iv) If $\left(y, y^{\prime}\right) \in R$ and $\left(y_{1}, y_{1}^{\prime}\right) \in R$ are mutually generic over $k$, then there is $z \in y * y_{1}$ such that $\left(z, y^{\prime} \cdot y_{1}^{\prime}\right) \in R$.

Proof. (i) is clear from Lemmas 3.3 and 3.1.

(ii) Suppose for a contradiction that $\left(y, y^{\prime}\right) \in R$ and $y \notin k\left(y^{\prime}\right)^{a l g}$. So $\operatorname{dim}\left(y, y^{\prime} / k\right)$ $>\operatorname{dim}\left(y^{\prime} / k\right)$. Let $\left(d, d^{\prime}\right)$ realize $p$, independently from $\left(y, y^{\prime}\right)$ over $k$. So

$$
\operatorname{dim}\left(y, y^{\prime}, d / k\right)>m+\operatorname{dim}\left(y^{\prime} / k\right) .
$$

Let $\left(d_{1}, d_{1}^{\prime}\right)$ be a realization of $p$ given by the definition above of $\left(y, y^{\prime}\right)$ being in $R$. Hence $\operatorname{dim}\left(y^{\prime}, d, d^{\prime}, d_{1}, d_{1}^{\prime} / k\right)=m+\operatorname{dim}\left(y^{\prime} / k\right)$. But $y \in k\left(d, d_{1}\right)^{a l g}$, so $\operatorname{dim}\left(y, y^{\prime}, d, d^{\prime}, d_{1}, d_{1}^{\prime} / k\right)=m+\operatorname{dim}\left(y^{\prime} / k\right)$, contradicting $(* *)$.

(iii) follows from Lemma 3.1.

(iv) Let $\left(y, y^{\prime}\right)$ and $\left(y_{1}, y_{1}^{\prime}\right)$ be mutually generic elements of $R$. This means that $\operatorname{dim}\left(y, y^{\prime}, y_{1}, y_{1}^{\prime} / k\right)=2 m$. Let $\left(d, d^{\prime}\right)$ realize $p$ independently of $\left(y, y^{\prime}, y_{1}, y_{1}^{\prime}\right)$ over $k$. Let $\left(d_{1}, d_{1}^{\prime}\right)$ be a realization of $p$ witnessing that $\left(y, y^{\prime}\right) \in R$ (namely $d_{1}^{\prime}=y^{\prime} \cdot d^{\prime}$, 
$d_{1} \in y * d$, etc.). Then $\left(d_{1}, d_{1}^{\prime}\right)$ is independent of $\left(y, y^{\prime}, y_{1}, y_{1}^{\prime}\right)$ over $k$, so again we find $\left(d_{2}, d_{2}^{\prime}\right)$ realizing $p$ witnessing that $\left(y_{1}, y_{1}^{\prime}\right) \in R$ (namely $y_{2}^{\prime}=y_{1}^{\prime} \cdot d_{1}^{\prime}$, etc.). It is easy to see that $\left(d, d^{\prime}\right)$ and $\left(d_{2}, d_{2}^{\prime}\right)$ are independent over $k$. Note that $\left(y_{1}^{\prime} \cdot y^{\prime}\right) \cdot d^{\prime}=d_{2}^{\prime}$. Also by associativity of $*$, there is $z \in y_{1} * y$ such that $d_{2} \in z * d$. The same argument as in the proof of Lemma 3.3 shows that $\left(z, y_{1}^{\prime} \cdot y^{\prime}\right)$ is independent from $\left(d, d^{\prime}\right)$ over $k$. Hence $\left(z, y_{1}^{\prime} \cdot y^{\prime}\right) \in R$, as required.

Lemma 3.4 finishes the proof of Proposition 1.1. Note that as $a_{2}, a_{2}^{\prime}$ are generic points of $X, G$ respectively over $k$, then part (i) of Lemma 3.4 gives that $R$ projects dominantly to both $X$ and $G$. Also it is immediate that $\left(e, e^{\prime}\right) \in R$.

\section{REFERENCES}

1. V. M. Buchstaber, n-valued groups: theory and applications, Moscow Math. Journal, vol. 6, no. 1 (2006), 57-84. MR2265947 (2007i:20102)

2. A. Pillay, Geometric Stability Theory, The Clarendon Press, Oxford Univ. Press, New York, 1996. MR.1429864 (98a:03049)

School of Mathematics, University of Leeds, Leeds LS2 9JT, United Kingdom

E-mail address: pillay@maths.leeds.ac.uk 